\title{
Contabilidad de costos y de gestión en la industria farmacéutica venezolana: Estudio de un caso*
}

\author{
Rincón de Parra, Haydée Cecilia**
}

\section{Resumen}

La competencia, los controles de precios, las políticas tributarias, la integración de los mercados latinoamericanos, la diversificación de los productos, entre otros aspectos, están llevando a las empresas a enfrentar condiciones cada vez más difíciles para su desarrollo y expansión. Ante este escenario, el estudio y análisis de los costos se convierte en un factor decisivo de la gestión empresarial. Con el presente trabajo se tiene como propósito indagar sobre las prácticas que realiza una empresa en el estado Mérida, en cuanto a contabilidad de costos y de gestión se refiere. La investigación corresponde a un estudio exploratorio-descriptivo. Se utilizaron como técnicas el análisis documental, la observación directa, la entrevista no estructurada y la encuesta. Los resultados revelan que la empresa no tiene establecidos los procedimientos adecuados que le permitan determinar y gestionar los costos de cada uno de los productos que elabora, por lo que se requiere que ésta fije estrategias para avanzar en el campo de la competitividad, administrarse en situaciones de escasez, participar activamente en el mercado nacional, en fin, utilizar herramientas que le permita introducirse en un mundo cada vez más interrelacionado en medio de cambios vertiginosos y en contextos turbulentos. Se concluye que es necesario que la empresa estudiada busque a lo interno la productividad y calidad apropiada.

Palabras clave: Contabilidad de costos, gestión de costos, calidad, industria farmacéutica.

Recibido: 04-04-12. Aceptado: 05-03-08

* Resultados del proyecto de investigación financiado por el Consejo de Desarrollo Científico, Humanístico y Tecnológico (CDCHT) de la Universidad de Los Andes (ULA).

** Participante del Doctorado en Ciencias Administrativas de la UNESR, Núcleo Caracas. Magíster en Gerencia de Empresas, Mención Industrial. Licenciada en Contaduría Pública. Profesora Titular de la Cátedra de Contabilidad de Costos de la Facultad de Ciencias Económicas y Sociales de la Universidad de Los Andes, Edo. Mérida. E-mail: haydocc@ yahoo.com. 


\title{
Accounting Costs and Management in the Venezuelan Farmaceutical Industry: A Case Study
}

\begin{abstract}
Competition, price controls, tax policies, the integration of Latin American markets, the diversification of products, among other things, are causing businesses to come up against increasing levels of difficulty for development and expansion. For this reason, the study and analysis of costs is becoming an ever more decisive factor in business management. The purpose of this study is to research the practices used by companies in Merida in relation to cost accounting and management. The research is exploratory and descriptive. The techniques of documentary analysis, direct observation, nonstructured interviews and surveys are used. The results reveal that business firms do not establish adequate procedures that allow for the determination and management of costs for each of the products they manufacture, and for this reason they need to establish strategies in order to improve competitiveness, administer situations of scarcity, participate actively in the national market, and to utilize tools that permit their introduction into an ever more interrelated world immersed in difficult changes and turbulent contexts. The conclusion is that it is necessary that for the companies studied to search for internal productivity and appropriate quality.
\end{abstract}

Key words: Accounting costs, cost management, quality, pharmaceutical industry.

\section{Introducción}

Hoy día las empresas se ven enfrentadas a situaciones competitivas, controles de precios y políticas tributarias. Aspectos éstos que tienen incidencia en la determinación de los márgenes de ganancia de las mismas.

El nuevo enfoque de gerencia consiste en la orientación de la empresa hacia el mercado, en donde ésta debe producir lo que pueda vender y no lo contrario; vender a toda costa lo que pueda producir. Más aún, ante la perspectiva de un consumidor de menor poder adquisitivo, más selectivo, la empresa está obligada a adaptarse a la real necesidad del mercado, antes de perder al consumidor $y / 0$ usuario de bienes y/o servicios.
Contextualizando se tiene que Venezuela se enfrenta a graves dilemas en materia económica, política y social, como son: severo déficit fiscal, gigantesca deuda interna, control de cambio, aumento desmedido de los precios y tarifas de los bienes y servicios, impuestos elevados, cierre de empresas y su incidencia en la creación de desempleo, poco desarrollo industrial, crisis generalizada de valores sociales, politización de las actividades corrupción en las estructuras de poder y concentración del poder político.

Esas circunstancias, más los dinámicos cambios tecnológicos y la integración de los mercados latinoamericanos, están afectando a las empresas del sector productivo y, entre éstas, a las empresas de la industria farmacéutica, y con 
mayor incidencia a las empresas de la industria de medicamentos genéricos, lo cual requiere que las mismas fijen estrategias de gestión y de costos, que les permitan avanzar y hacerlas más competitivas, y puedan incorporarse al acelerado crecimiento tecnológico, administrarse en situaciones de escasez, participar activamente en los mercados nacionales e internacionales, en fin, aprender a trabajar en un mundo cada vez más interrelacionado caracterizado por cambios vertiginosos y contextos turbulentos. Es esta situación, lo que llevó a realizar la presente investigación, que tiene como objetivos fundamentales indagar sobre las prácticas que realiza la empresa PROULA MEDICAMENTOS, C. A., en cuanto a contabilidad de costos y de gestión; así como también, proponer algunas estrategias orientadas hacia la mejora de la calidad, la competitividad y la excelencia empresarial.

La investigación se justifica en los términos siguientes: a) La relevancia socialque tiene, por cuanto el estudio de los costos se orienta hacia la optimización de los mismos y, por consiguiente, se pueden ofrecer productos a bajo costo y de excelente calidad. b) Desde el punto de vista institucional, dado que, a través del estudio se pueden establecer parámetros que permitan resolver en la planta de medicamentos, problemas de determinación, análisis, control y reducción de costos de producción, en la búsqueda de la mejora continua de los procesos de producción, con miras a ser más competitivos. Por otra parte, como el estudio está inserto dentro de la línea de investigación "Las Ciencias Contables y Financieras: su epistemología y aplicabilidad" y de la sub línea "Contabilidad de Costos y de Gestión" del Centro de Investigación y Desarrollo Empresarial de la Facultad de Ciencias Económicas y Sociales de la Universidad de los Andes, ésta servirá para alimentar la investigación que se desarrolla en la misma, pues, existen pocos trabajos sobre este eje temático, por lo tanto, el estudio servirá como marco de referencia para posteriores investigaciones en el área. Además, da la oportunidad al investigador de convivir con el quehacer empresarial y hacer de la praxis docente-investigativa una actividad más realista y menos teórica.

Desde el punto de vista metodológico, la investigación es de tipo exploratoria-descriptiva y, en su diseño, se enmarca en las modalidades documental y de campo.

El estudio está centrado en una muestra intencional, es decir, en la empresa PROULA MEDICAMENTOS, C. A., ubicada en el Sector Industrial La Alegría, La Variante, Lagunillas-Estado Mérida.

Las técnicas de recolección de la información se fundamentan sobre el análisis documental, la observación directa de los procesos de la empresa (se tuvo el cuidado de no intervenir en los mismos, bajo ningún pretexto), la entrevista no estructurada y la encuesta que se aplicó a los responsables de manejar la información relacionada con la contabilidad y gestión de los costos. Esta actividad la realizó el investigador de manera personal, lo que le permitió involucrarse con el objeto investigado y garantizar la confidencialidad de la información. El uso de la técnica de la entrevista facilitó la labor de persuasión en el entrevistado, cuando mostró resistencia a dar la infor- 
mación requerida, estableció la posibilidad de verificar respuestas de los cuestionarios, además, permitió ampliar la información suministrada en los mismos y ver la reacción de los entrevistados; así como recoger expresiones que fueron de interés para el análisis de la investigación. La información recolectada se organizó, procesó y presentó de manera sencilla, ordenada y clara, mediante datos condensados en cuadros que permitió el análisis e interpretación de los mismos. En la interpretación de la información se trató de encontrar un significado más completo y amplio de la información empírica recabada. En primer lugar, se analizaron e interpretaron los datos por separado, luego se realizó una síntesis de los resultados para dar explicación al problema investigado y, por último, se procedió a realizar una síntesis general que permitió llegar a conclusiones con relación a la problemática planteada.

\section{Sistemas de contabilidad de costos y de gestión}

Kaplan y Cooper (1999) señalan que los sistemas de contabilidad de costos y de gestión son útiles, entre otros aspectos, para: a) diseñar productos (bienes y servicios) que satisfagan las expectativas de los clientes y, al mismo tiempo, puedan ser producidos y entregados con una utilidad; b) detectar dónde hay que realizar mejoras continuas o reingeniería de calidad; c) guiar las decisiones de inversión y oferta de productos; d) negociar con los clientes el precio, las características del producto, la calidad, las condiciones de entrega y el servicio a satisfacer; e) estructurar procesos eficientes y efica- ces de distribución y servicio para los segmentos objetivo de mercados y de clientes.

La Contabilidad de Costos es un sistema de información que se centra en la determinación de los costos de los productos (bienes o servicios) que ofrece la organización, relacionándolos con los ingresos obtenidos por la venta de los mismos, generando así, información para la toma de decisiones.

Los costos de los productos, para efectos de presentar los reportes financieros externos, son de dos tipos: "producción y no producción" (Hansen y Mowen, 1996: 41). Los costos de producción están asociados con la manufactura (fabricación) de bienes o la provisión de servicios, y son: los materiales directos, la mano de obra directa y los costos indirectos de fabricación. Los materiales directos "son los que pueden ser identificados con determinados pedidos de producción o con una determinada unidad que constituye una parte del producto acabado... y pasan directamente a formar parte integrante del producto, de tal manera que representa un porcentaje alto de su costo" (Mallo et al., 2000: 119). Es decir, el costo de estos materiales se carga directamente a los productos, dado que es fácil medir y cuantificar la cantidad consumida por producto. Ejemplo, los principios activos y excipientes que conforman un medicamento. La mano de obra directa es la que transforma la materia prima (materiales directos) en otro producto tangible, o la que proporciona un servicio a un cliente. El costo de esta mano de obra se imputa directamente al producto, debido a que es fácil determinar y cuantificar el tiempo trabajado en 
cada producto o lote de productos. Ejemplo, los costos asociados con los trabajadores que intervienen en el proceso de mezclado y encapsulado en la fabricación de cápsulas, en la línea de producción de antibióticos. Los costos indirectos de fabricación (CIF) están referidos a todos los costos de producción distintos de los materiales directos y de la mano de obra directa. Estos costos son: a) los costos de los materiales indirectos, los cuales son utilizados en las operaciones de fabricación; y aunque algunas veces pasan a formar parte del producto, su consumo no puede ser identificado de una manera precisa con las órdenes de producción o su valor es ínfimo con respecto al costo total del producto, b) Los costos de mano de obra indirecta, que no se relacionan de manera específica con el producto obtenido, por lo tanto no se cargan de manera directa a dicho producto y c) Otros costos indirectos como son: servicios públicos, reparaciones de las maquinarias, depreciación de las maquinarias, seguro de la fábrica, alquiler de la fábrica, impuestos de la fábrica, etc. Los costos indirectos de fabricación no se cargan de manera directa a los productos, sino a través de diversos criterios de imputación, basados en las características específicas de las empresas.

Por lo general, existen dos criterios para asignar los costos indirectos de fabricación: un primer criterio que se fundamenta en el hecho de que los productos consumen los recursos en proporción a su volumen de producción, el cual puede ser expresado en variables físicas (horas de mano de obra directa, horas máquinas y unidades producidas) y en variables financieras (costo de materiales directos y costo de mano de obra directa) (Rincón, 2000). La asignación de los CIF a los productos se hace a través de una tasa predeterminada, que puede ser única para toda la planta o departamental, calculadas sobre la base del volumen de producción.

El segundo criterio se orienta hacia el costo de los componentes y no de los productos. Por un lado, se considera que los productos no consumen costos, sino que los mismos consumen actividades, por otro lado, las actividades son las que consumen recursos, por ello los costos se convierten en la expresión cuantificada en términos monetarios de esos recursos o factores productivos consumidos por las actividades. En consecuencia, son las actividades y no los recursos los que generan costos (Sáez; Fernández y Gutiérrez, 1993). La asignación de los CIF a los productos se hace a través de la determinación de los costos de las actividades.

\section{Los costos de no producción es-} tán asociados con las funciones de ventas y administración. Los costos de ventas, comerciales o de mercadotecnia como también se les denomina, son los costos necesarios para comercializar y distribuir un producto tangible o servicio. Como ejemplo de estos costos se tienen los sueldos y comisiones del personal de ventas, la publicidad y promoción, envíos y servicios a los clientes. Los costos administrativos son los costos de la administración general de la organización, es decir los costos en que se incurren para asegurar que las diversas actividades de la organización estén integradas apropiadamente para alcanzar la misión global de la empresa. Para efectos de preparar los reportes financieros externos, estos 
costos de no producción, se consideran no inventariables, es decir, costos del período "que no se adicionan al valor de los productos elaborados, sino que se cargan a los ingresos del período de una manera directa, inmediata y en su totalidad" (Rincón, 2001: 57).

Para calcular, acumular y analizar los costos de los productos tangibles o servicios, se manejan tres tipos de sistemas de costos: a) sistemas de costos por órdenes de producción, b) sistema de costos por procesos de producción y c) sistema de costos por operaciones (híbrido o intermedio).

El sistema de costos por órdenes de producción, según (Hansen y Mowen, 1996), presenta las características siguientes: a) lo utilizan las empresas que elaboran una gran variedad de productos o trabajos que, por lo general, son muy distintos unos de otros. b) En este sentido, los costos de cada uno de los trabajos son diferentes, y se deben seguir y acumular por separado, a través de un documento que es la $<<$ hoja de costos de la orden de producción $>>$ y c) Cuando se termina un trabajo, el costo unitario se obtiene dividiendo los costos totales de fabricación entre la cantidad de unidades producidas.

Según estos últimos autores mencionados, un sistema de costos por procesos de producción se caracteriza por contar con un gran número de productos homogéneos que pasan por una serie de procesos. En cada proceso o centro de costos pueden necesitarse insumos de materiales, mano de obra y costos indirectos, y al completarse un proceso, la producción parcialmente terminada se transfiere a otro proceso, de tal manera que los costos se acumulan por procesos o centros de costos para un determinado período, a través de un documento que es el <<informe de recepción>>. Los costos unitarios se calculan promediando los costos totales del proceso del período entre la producción del mismo.

El sistema de costos por operaciones es viable para las empresas que elaboran diferentes productos, utilizando distintos tipos de materiales directos, pero con operaciones similares de procesamiento (Gayle, 1999).

Para asignar los costos se utilizan dos sistemas de costos: a) Modelo de costo completo o por absorción (full costing) y b) Modelo de costo variable o directo (direct costing). A través del sistema de costo completo, en cada fase de producción, los productos van absorbiendo todos los costos (fijos y variables) relacionados con su fabricación. En el sistema de costo variable, se le imputa al producto aquellos costos de producción que varían según el volumen de fabricación, y los costos fijos, cuyo consumo no tenga una relación directa con el volumen fabricado, no serán incorporado al costo de producción y se considerarán un costo del período.

La Contabilidad de Gestión "tiene como principal objetivo facilitar a la dirección información útil para la toma de decisiones económicas a través de la planificación y el control de gestión" (Mallo et al., 1994: 9), así como dar respuesta a las necesidades planteadas en el seno de las organizaciones.

En este sentido, la contabilidad de gestión se orienta, entre otros aspectos, a transmitir a todas las personas involucradas en la organización, la información 
necesaria para la gestión, por ejemplo: costos, rentabilidad e indicadores no monetarios. También dirigir todos los esfuerzos de la organización hacia la consecución de los objetivos, procurando la eficiencia y la eficacia en la utilización de los recursos, para ello, se utilizan los presupuestos, los análisis de desviaciones y la toma de medidas correctivas. Asimismo, debe contribuir a que todos los responsables se sientan motivados para la consecución de sus objetivos (Amat y Soldevilla, 1998).

En este mismo orden de ideas, Fernández (1994) señala que a través de la contabilidad de gestión lo que se busca es proporcionar información tanto a largo como a corto plazo referida al ámbito interno y externo de la empresa, adecuada para alcanzar ventajas competitivas. A este respecto, la información de carácter no financiero, tanto cualitativa como cuantitativamente, adquiere una gran significación. Considera también este autor que la información podrá ir dirigida a cualquier estamento de la organización, y no sólo a la alta gerencia, tal como tradicionalmente se ha venido haciendo.

Cabe señalar que la contabilidad de gestión comprende una serie de herramientas relacionadas con el enfoque de proceso de negocio. Algunas de ellas son: a) costos y gerencia basada en las actividades (ABC y ABM, iniciales de $A C$ tivity Based Costing y Activity Based Management); b) costo meta o costo objetivo (target costing); c) justo a tiempo (just in time); d) gestión de la calidad, e) gestión de costos de marketing, y f) costo y gestión medioambiental.

Para Holst y Savage (2002) el costo basado en las actividades $(\mathrm{ABC})$ y la gerencia basada en las actividades (ABM) se han considerado muy importantes en la década de 1990 y continuarán creciendo rápidamente en términos de utilidad y aplicación en el siglo XXI, como sistemas efectivos para determinar y controlar costos, incrementar las utilidades, y para ayudar a mejorar el desempeño en los negocios. En este sentido, Rincón (2000: 24) señala que a través del costo y la gerencia basados en las actividades "se busca calcular costos más exactos, controlar costos y mejorar la eficiencia, lo cual consiste en gerenciar la empresa mediante el dominio de sus actividades y procesos". Por lo tanto, lo importante no es el costo del producto, sino el costo de las actividades que conforman el mismo.

El costo basado en las actividades (ABC) se define como un método que permite asignar, en primer lugar, el costo de los recursos a las actividades, mediante los impulsores de costos, y luego el costo de esas actividades a los objetos de costos, a través de los impulsores de actividades. Un objeto de costo puede ser un producto, un proceso, un centro de costos, una línea de producción, un servicio, un cliente, entre otros.

Sobre este particular, Mallo et al (1994:470) definen el ABC como un sistema "que pretende asignar los costos directos y distribuir los costos indirectos sobre el costo del producto... Los costos deben ser distribuidos a través de las verdaderas causas que generan estos costos. Las causas de los costos se identifican con las actividades necesarias para su elaboración y venta, siendo las principales clasificaciones de éstas, entre otras: 1) diseño del producto o servicio, 2) ingeniería, 3) fabricación, 4) comercializa- 
ción, 5) envío, 6) facturación, y 7) servicio postventa".

Al respecto, Álvarez et al (1996) argumentan que en el sistema de costos por actividades, la estructura de medición de los costos se proyecta sobre el área de producción y sobre las etapas anterior y posterior a la fabricación del producto, abarcando por lo tanto las etapas de diseño, lanzamiento, seguimiento y servicio postventa de un producto.

En resumen, el costo basado en las actividades se caracteriza por: a) ser una metodología que mide los costos, las actividades, los recursos y los objetos de costo, y porque puede plantearse en términos de costos históricos y costos estándar. b) Ser aplicable a todo tipo de organizaciones: industrial, comercial y de servicios. c) Trabajar en función de las relaciones causales entre los inductores de costos y las actividades, debido a que para cada causal de costos existe un marco individual de análisis de costos, que es crucial para entender el posicionamiento de una empresa (Shank y Govindarajan, 1995). d) Permitir la identificación, descripción, clasificación y análisis de las actividades, a nivel unitario, de lote y de línea. e) Lograr la transformación de la mayoría de los costos indirectos respectos a los productos, en costos directos respecto a las actividades. f) Situarse muy bien en los nuevos enfoques gerenciales de justo a tiempo, calidad total, planificación de los recursos de la producción y otros. g) Lograr una mejor determinación del costo de los productos en aquellas empresas que se muevan en un ambiente muy competitivo; tengan una gran variedad y/o complejidad de productos; un elevado número de clientes y ca- nales de distribución; una variedad de procesos de producción; los costos indirectos de fabricación y los costos del período, sean una parte importante de los costos totales de la empresa, y los mismos se imputen a los productos de manera arbitraria.

\section{La gerencia basada en las activi-}

dades (ABM) es una evolución del costo basado en las actividades. El ABM está considerado como "el proceso de gestión mediante el cual la información por el ABC permite a los gestores conocer las actividades y los procesos de negocios, dando una visión más integral y horizontal de la empresa" (De la Cuesta, 1996: 217), por lo tanto, el ABM tiene una proyección orientada a la gestión de la empresa en general, con el objetivo de conseguir la excelencia empresarial.

Plantean Kaplan y Cooper (1999) que el ABM permite a la organización alcanzar sus resultados con menos recursos, a través de dos subsistemas complementarios: el ABM operativo y el estratégico. El ABM operativo, orientado a desarrollar las acciones correctamente, ayuda a aumentar la eficiencia, reducir los costos y optimizar la utilización de los activos, reduciendo el tiempo de paro de las máquinas, mejorando o incluso eliminando las actividades y procesos defectuosos. El $A B M$ estratégico, orientado a desarrollar las acciones correctas, permite elegir las actividades que se deben realizar. Engloba decisiones respecto al diseño y desarrollo del producto, tipo de clientes y relaciones con éstos, relaciones con los proveedores, segmentación del mercado y canales de distribución, entre otras.

El ABM, que tiene como propósito ayudar a las organizaciones a crear más 
valor para sus clientes, al tiempo que reduce el costo de las operaciones, se caracteriza por: 1) Orientar la gestión hacia aquello que satisfaga a los clientes internos y externos, permitiendo que sean ellos los que determinen las actividades a realizar. 2) Analizar las actividades que configuran la cadena de valor de la empresa, posibilitando la eliminación de actividades que no generan valor añadido, sin que tenga un impacto negativo en el producto tangible o servicio pagado por el cliente o en el cumplimiento de requisitos externos, dado que no se trata de hacer mejor lo que es suprimible; optimizar las actividades que si bien no agregan valor, no se pueden eliminar por ser necesarias en el proceso; y mejorar de forma continua las actividades con valor añadido, las cuales se caracterizan por intervenir en gran medida en los atributos del producto, creando así una cultura donde todos se preocupen por ser más eficientes. 3) Crear y mantener un ambiente de mejoramiento continuo, bajo la idea de que siempre existirá una mejor manera de hacer las cosas. 4) Dirigir los procesos a través de la gestión de las actividades que se realizan en la empresa y no mediante la gestión de los costos, es decir, permitir el control de lo que se hace en lugar de lo que se gasta.

En cuanto al Target Costing, considerando lo planteado por Blanco (1996) y Hansen y Mowen (1996), se señala lo siguiente: cuando las empresas operaban en un mercado estable, la forma de determinar el precio de venta estuvo basado en la ecuación: Costo + Utilidad = Precio de Venta, en donde por consecuencia, los precios eran determinados generalmente por los costos de produc- ción, convirtiéndose el precio en la variable dependiente. Más tarde, con la apertura económica de los años noventa, la competencia tanto interna como externa y la disminución de la vida útil de los productos originada por los avances en la tecnología, produjo cambios en el enfoque del precio con respecto al costo, en cuanto a que éste pasa a ser una función dependiente del precio y de la utilidad deseada o planeada, es decir, el precio de venta constituye el punto de partida, suponiendo una clara orientación hacia el cliente, lo que lleva a que la fórmula anterior se convierta en: Costo Planeado = Precio de Venta Planeado - Utilidad Planeada. Es este nuevo enfoque del costo lo que se denomina target costing.

El target costing (traducido como costo objetivo, meta, planeado o proyectado) lo desarrolló la Organización Toyota en Japón en la década de los sesenta, y sus orígenes se encuentran en la ingeniería de valor. Según Monden (1997: 217), la Japan Value Engineering Association define a la ingeniería del valor como "los esfuerzos organizados para realizar análisis funcionales de productos/servicios para lograr de forma fiable, todas las funciones requeridas al costo del ciclo de vida más bajo posible". En este sentido, el target costing no es una simple técnica de cálculo de costos, sino que implica un mecanismo integrado de gestión de costos para unir de manera coherente las distintas áreas funcionales de la empresa, que permita lograr un nivel de utilidad acorde con los objetivos prefijados. Por tanto, en la empresa debe existir una relación permanente entre los investigadores de mercados, los diseñadores del producto, el personal de fabri- 
cación y comercialización, y el analista de costo.

El costo objetivo se conceptualiza como una herramienta de gestión de costos orientada a reducir el costo total de un producto a lo largo de todo su ciclo de vida, con la ayuda de las áreas de producción, ingeniería, investigación y desarroIlo, marketing y finanzas (Sakurai, 1989). Para implantar el target costing en una organización, es necesario partir de una visión global de los costos del producto, dado que en este nuevo enfoque se consideran los costos generados en todas las etapas del ciclo de vida del producto. En este sentido, las empresas, para lograr nuevos mercados o penetrar en determinados nichos de mercados, deben garantizar la calidad total de los productos, en términos de satisfacer las necesidades de los clientes; así como ofrecer un precio que asegure la demanda, pues los precios son impuestos por el mercado. Ese precio debe permitirles un beneficio adecuado, así como determinar el costo máximo en que pueden incurrir -nivel deseado de costos- (Rincón, 2000). Por otra parte, implantar el método del target costing exige un compromiso total de la organización y mucho esfuerzo de los miembros de la misma, así como una visión permanente de mejora continua y reducción sistémica de costos incorporada en todos los sectores, dado que este método está inserto en la filosofía del ciclo de vida del producto y en la filosofía de orientación al mercado y al cliente.

El enfoque $<$ Just In Time $>$ traducido a Justo A Tiempo (JAT) "es una filosofía industrial, de eliminación de todo lo que implique desperdicio en el proceso de producción, desde las compras hasta la distribución" (Hay, 1989: 1). Para este autor, la eliminación del desperdicio tiene como resultado un proceso orientado a la calidad y capaz de responder a las necesidades del cliente en el menor tiempo posible.

En consonancia con estas ideas, Tanaka et al (1997: 141) argumentan que el enfoque justo a tiempo de la compra y la producción proporciona "una filosofía y una práctica de adquisición dirigida por la demanda y el uso de recursos". En este contexto, las empresas no deben mantener inventarios, dado que los mismos se consideran desperdicios. Si tomamos en cuenta que el objetivo fundamental del JAT es la reducción o eliminación de inventarios, una empresa que se organice bajo esta filosofía, eliminaría los riesgos y costos asociados con el mantenimiento de éstos, como son: el riesgo de dañar las existencias, el riesgo de que los stocks queden obsoletos, el costo de oportunidad de tener fondos inmovilizados en stocks, y el costo de almacenar, mantener y mover internamente los stocks (Tanaka et al., 1997:141). Con esta nueva manera de administrar los inventarios se lograría "por un lado, aumentar las utilidades al eliminar los costos que generan los inventarios innecesarios y por otro lado, mejorar la posición competitiva de la empresa al incrementar la calidad y flexibilidad en la entrega a los clientes" (Ramírez, 1997: 118).

En resumen, la filosofía JAT, más que una técnica para reducir el costo de los inventarios, se considera como una herramienta de gestión que impulsa la mejora de todo el proceso de producción, eliminando todas aquellas actividades que no agregan valor y originando efec- 
tos positivos en los resultados y en la competitividad de la empresa.

Con respecto a la calidad, se puede señalar que es un concepto que ha evolucionado considerablemente durante las últimas cuatro décadas. A juicio de Besterfield (1995:1) "el término calidad, por lo general lo asociamos con productos o servicios excelentes, que satisfacen nuestras expectativas y, más aún, las rebasan". Por su parte, Campanella (1992: 63) señala que "calidad quiere decir conformidad con los planos y especificaciones y el control de calidad determina si el producto es conforme... Calidad se utiliza en un sentido más amplio y significa adecuación para el uso por parte del usuario... y abarca tanto la calidad de diseño, como la calidad de conformidad" . La Asociación Americana de Control de Calidad (ASQC, en sus siglas del inglés American Society for Quality Control), define la calidad como la totalidad de prestaciones y características de un producto o servicio que son la base de su capacidad para satisfacer necesidades explícitas o implícitas.

Se habla de calidad como conformidad, como satisfacción de las expectativas del cliente, como valor con relación al precio, como excelencia (Moreno; Peris y González, 2001).

La gestión de la calidad se ha producido en cuatro fases: inspección, control de calidad, aseguramiento de la calidad (AC) y gestión de la calidad total (GCT) (Moreno; Peris y González, 2001). La inspección y el control de calidad están orientados a resolver problemas de tipo operativo y tienen escasa influencia en la dirección, mientras que el AC y la GCT constituyen los dos grandes enfo- ques de dirección y, dada la visión cada vez más global que se tiene de la gestión de la calidad, éstos abarcan a la inspección y el control de calidad.

La identificación y medición de los costos relacionados con la calidad se hace imprescindible porque, según (Horngren, Foster y Datar, 1996:794) los mismos "varían de 15 a $20 \%$ de los ingresos de ventas de muchas organizaciones" y porque, siguiendo a Dale y Plunkett (1993: 5), permiten: a) que la calidad se considere como uno de los factores variables de los negocios, al igual que, por ejemplo el marketing, la investigación y el desarrollo, la producción y las operaciones. b) Subrayar la importancia de la calidad de los productos y servicios para la buena marcha de la empresa. c) Influir sobre el comportamiento y las actitudes de los empleados de todos los niveles de la empresa, con respecto a la GCT y el mejoramiento continuo de la calidad. d) Centrar la atención en asuntos en los que se gastan grandes cantidades, y detectar las oportunidades que en potencia podrían ayudar a reducir costos. e) Medir el desempeño y constituir una base para la comparación interna entre productos, servicios, procesos y departamentos.

Según esos autores los costos de la calidad incluyen "los costos originados en el diseño, la implantación, la operación y el mantenimiento del sistema de calidad, así como los costos por fallas en el sistema, los productos y el servicio". Estos costos se clasifican en cuatro categorías, a saber: costos de prevención, costos de evaluación, costos por defectos internos y costos por defectos externos. Los costos de prevención son los cometidos por todas las actividades diseñadas 
específicamente para detectar o prevenir la mala calidad de los productos o servicios. Se incurre en los costos de evaluación para determinar si los productos y servicios cumplen con los estándares de calidad y requisitos de funcionamiento. Los costos por defectos internos son los que causa la empresa, cuando no logra la calidad específica antes de ser enviados al cliente. Las mayores partidas de costos son las de los desperdicios y la reelaboración. Se definen como costos por defectos externos aquellos en los que incurre la empresa cuando no logra la calidad específica después de ser enviados al cliente.

En cuanto al marketing, hay que señalar que éste es algo más que el simple proceso de venta y publicidad. Se considera que su papel es esencial en el mecanismo de ofrecer al público bienes y servicios que satisfagan sus necesidades y abarca diversas áreas, tales como: ventas, publicidad, dirección de proyectos de investigación de mercados, distribución, servicios al clientes y otras. Así, el concepto de marketing "implica que una empresa dirige todas sus actividades a satisfacer a sus clientes, y al hacerlo obtiene un beneficio" (McCarthy y Perreault, 1996: 36). Esta definición contiene tres ideas fundamentales. La primera tiene que ver con que la satisfacción del cliente se orienta a dirigir el sistema completo (producción, finanzas, ventas, recursos humanos). La segunda a propiciar un esfuerzo global de la organización (trabajar en equipo para realizar un trabajo mejor) y la tercera en la obtención de un beneficio -no simplemente las ventas- como objetivo.

El concepto de marketing no se desarrolla sin un costo. El análisis y gestión de costos de las actividades mercadológi- cas es de importancia, y por lo tanto el marketing no debe ser considerado meramente como un gasto general. Los costos de marketing incluyen, entre otros, las promociones de ventas y la publicidad, la distribución física, la investigación de mercado, el desarrollo del producto y el servicio al cliente. A estos costos, en la actualidad, se les está dando una mayor importancia debido a que los mismos pueden representar parte significativa de los costos totales. De hecho, en algunas empresas los costos de marketing son mayores a los costos de producción. El estudio y análisis de los costos de marketing se considera una herramienta de gestión muy valiosa en las empresas, dado que permite dar respuesta a las siguientes interrogantes: ¿Qué clientes producen utilidades? ¿Qué producto resulta más redituable vender? ¿En qué zonas se hacen las ventas que producen más utilidades?, ¿Qué vendedores están realizando las ventas que producen las mayores utilidades? (Neuner y Deakin, 1997).

En cuanto a la problemática ambiental, en las últimas dos décadas ha habido una preocupación creciente que ha llevado a los gobiernos y otras entidades, incluyendo la Organización de las Naciones Unidas (ONU), a desarrollar instrumentos de diversa naturaleza, con el fin de proteger y restaurar el medio ambiente, al menor costo para la sociedad.

Una gran mayoría de empresas (mineras, petroleras, alimenticias, farmacéuticas, siderúrgicas, químicas, entre otras) que desarrollan actividades con tendencias a contaminar el medio ambiente, han establecido políticas y estándares de calidad ambiental (Suárez, 2001), como medidas para reducir el 
daño ambiental y, simultáneamente, mejorar su posición competitiva.

Los costos que se derivan de una estrategia $o$ acciones tendentes a una gestión medioambiental, están asociados con la clasificación de costos ambientales, presentada por la Asociación Española de Contabilidad y Administración de Empresas (AECA): i) costos medioambientales recurrentes (costos derivados de la obtención de información medioambiental, costos derivados de un plan de gestión medioambiental, costos derivados de la adecuación tecnológica medioambiental, costos derivados de la gestión de residuos, emisiones y efluentes, costos derivados de las exigencias administrativas y costos derivados de la auditoría medioambiental). ii) Costos medioambientales no recurrentes (costos derivados de los sistemas de información y prevención medioambiental, costos derivados de las inversiones en instalaciones, costos plurianuales de conservación y mantenimiento, costos derivados de la interrupción del proceso, costos derivados de accidentes, costos derivados de las nuevas exigencias del entorno, costos derivados de la mejora de la imagen medioambiental y costos de los sistemas de control y medición).

\section{Características generales de PROULA}

Los resultados y el análisis de la información se presentan considerando, en conjunto, los objetivos específicos planteados en la investigación, la información obtenida en los cuestionarios y las opiniones emitidas por los gerentes medios y jefes de departamentos de la empresa objeto de estudio.
PROULA Medicamentos, C.A. es una empresa farmacéutica de la Universidad de los Andes fundada en 1991 con el objeto de desarrollar, producir y comercializar medicamentos genéricos y de marca. En 1994 comienza sus operaciones, y las actividades desarrolladas durante ese año, se consideraron como un proceso de prueba y arranque. Desde 1995, con una moderna planta ubicada en Lagunillas, Municipio Sucre del Estado Mérida, participa activamente en el mercado nacional. A partir de Agosto de 2001, por sus rigurosas normas de producción, calidad y sentido ético en el desempeño profesional, recibe la Certificación de Buenas Prácticas de Manufactura (BPM), otorgado por la División de Drogas, Medicamentos y Cosméticos del Ministerio de Salud y Desarrollo Social, cuya vigencia es de dos años.

PROULA Medicamentos es la única empresa farmacéutica de carácter universitario, formalmente constituida en Venezuela. Es una compañía anónima, cuyo accionista mayoritario es la Universidad de los Andes y opera bajo la responsabilidad de una Junta Directiva que se rige por el Código de Comercio y los Estatutos de la empresa. En la actualidad, cuenta con un capital social suscrito de 42 millones de bolívares pagado $100 \%$. Las principales fuentes de financiamiento son la reinversión de utilidades que abarca $80 \%$, y $20 \%$ representado por crédito a corto plazo de proveedores. Cabe señalar que PROULA Medicamentos no realiza actividades de exportación, pero sí actividades de importación, que representan $50 \%$ del volumen total de compras del negocio. En cuanto a su estructura organizativa, hay que señalar 
que es piramidal, con líneas de mando definidas, en donde los responsables de cada unidad organizativa conocen sus funciones y responsabilidades, así como las del personal a su cargo.

Desde el punto de vista corporativo, PROULA Medicamentos se rige por los principios éticos y filosóficos que a continuación se señalan: a) Mantenimiento de estrictas normas de calidad y desempeño en la producción de medicamentos farmacéuticos mediante instalaciones, equipos y suministros acordes a las normas internacionales. b) Alta sensibilidad social en las dinámicas de comercialización, mercadeo, promoción y ventas, para facilitar productos medicinales de costo asequible para los venezolanos. c) Adopción de técnicas productivas de bajo impacto ambiental, propiciando un acercamiento al cumplimiento de las normas ambientales. d) Respeto por la vida y los estándares de bioética humana y social. e) Desempeño e interacción social a favor de los recursos naturales y el medio ambiente, centrando esfuerzos en la región sede. e) Estímulo constante al esfuerzo creador y laboral en el ámbito interno, apoyando la meritocracia y avalando acciones afines con la responsabilidad y el compromiso. f) Apoyo a la investigación científica y tecnológica en el sector salud, dentro y fuera del ámbito universitario y nacional.

Sobre la base de lo planteado en el párrafo anterior, la empresa ha definido su visión y misión en los términos siguientes: "Visión: competir y lograr lugares de liderazgo en escenarios nacionales como empresa farmacéutica de la Universidad de los Andes en la producción y comercialización de medicamentos, productos naturales y cosméticos -de excelente ca- lidad- apegada a principios y valores éticos a favor de la salud y economía de la sociedad venezolana. Misión: mediante una gestión empresarial autónoma, investigar, desarrollar, producir, distribuir y comercializar productos farmacéuticos, naturales y cosméticos -de excelente calidad- para satisfacer las necesidades de nuestros clientes".

En relación con los productos que elabora, la empresa dispone de 138 fórmulas registradas. En estos momentos, basados en actualizadas investigaciones de mercado, distribuye 42 fórmulas de alta calidad, a través de 52 presentaciones con amplia aceptación en el mercado nacional. La producción se orienta hacia tres líneas de productos: sólidos, líquidos y antibióticos no estériles, y tiene, como complemento de esas líneas, un proyecto para una nueva línea de productos inyectables (antibióticos). Además, están trabajando para concretar el proyecto de fabricación de antirretrovirales, productos que en Venezuela no se fabrican, sino que el Ministerio de Salud y Desarrollo Social los compra a las transnacionales a unos costos muy elevados y los suministra gratuitamente a los pacientes con VHI/Sida.

\section{Estructura del costo de producción}

Del cuestionario aplicado en la empresa, se obtuvo la siguiente información:

A los productos se les imputa la totalidad de los costos (fijos y variables), por lo que el método de cálculo de costos, según sus elementos, es el sistema de costeo por absorción y no el sistema de costeo directo o variable. Se trabaja con presupuestos sobre la base de los datos 
históricos y el comportamiento de la demanda, en tal sentido, el método de cálculo de costos, de acuerdo con la fecha de cálculo, es el sistema de costos estimados y no el sistema de costos estándar. Se trabaja con lotes de producción estándar, es decir, un número de unidades a producir uniforme para los distintos tipos de productos que se elaboran en las diferentes líneas de producción.

A través del cálculo de los costos, obtienen información para planificar, controlar, fijar precios, preparar estados financieros y tomar decisiones. Sin embargo, cabe preguntarse ¿cómo pueden planificar, controlar, fijar precios y tomar decisiones, si sólo manejan información detallada de la cantidad y costos de materia prima y material de empaque que se incorpora al producto? No manejan información, por ejemplo, de costos por concepto de mano de obra imputado a cada producto y línea de producto, así como tampoco de los costos indirectos de fabricación reales, ni costos indirectos de fabricación aplicados a cada producto y línea de producto. En resumen, no manejan información de costo por producto y línea de producción. Sólo manejan información de costos de manera global.

Si la empresa sólo conoce costos globales de todo el personal $(29,5 \%$ ) y de los cuales $5,8 \%$ se corresponde con la mano de obra directa. Cabría preguntarse, ¿cómo determinan ese porcentaje de costos directos?, dado que si no manejan información de los tiempos que labora cada trabajador en las líneas de producción y, por consiguiente, en cada tipo de producto, no pueden imputar costos de mano de obra a cada uno de ellos y ser considerados como costos directos. La asignación de los costos por concepto de mano de obra a los productos, sobre la base de un porcentaje, tal como se realiza en la empresa, indica que esos costos no pueden ser considerados como directos, sino como indirectos. Pareciera que no se tiene una clara concepción de lo que implica el término "directo" en el proceso de imputación de los costos a los productos y líneas de productos.

Los costos totales de materiales representan $34,3 \%$ y de éstos, $33,6 \%$ son materiales directos, de allí que el mayor control se ejerza sobre los mismos.

No manejan informe de las distintas partidas que conforman los costos indirectos de fabricación, sólo conocen los costos por concepto de depreciación de la maquinaria, la representa $4,23 \%$ del costo total de producción.

En la empresa utilizan una tasa predeterminada única para aplicar los costos indirectos de fabricación y emplean como base de aplicación, los costos por horas-hombres. Cabe preguntarse de nuevo, ¿si no manejan detalle de los tiempos trabajados en la elaboración de cada tipo de producto, entonces, cómo pueden calcular los costos por horashombres? y ¿cómo determinan, entonces, la tasa de aplicación única?

\section{Nuevos sistemas y factores claves de gestión}

En la empresa se conoce, pero no se utiliza, el sistema de costos basado en las actividades (ABC). De igual forma, no conocen el sistema de gerencia basada en las actividades (ABM). En la empresa se han realizado "estudios de las tareas ejecutadas, se han eliminado actividades 
que no agregan valor, se han racionalizado actividades que son necesarias y que no agregan valor, y se toman en cuenta los requerimientos del cliente para cambiar las actividades necesarias para satisfacerlos". Lo que nos lleva a preguntarnos, ¿si existe un desconocimiento del contenido que engloba el término ABM, cómo puede la empresa realizar tareas como las descritas entre comillas, las cuales son inherentes a la gerencia basada en las actividades? En la empresa se interesan por la posición de sus competidores, con el propósito de mejorar la imagen, reorganizar las tareas internas y mejorar la posición competitiva.

La empresa no trabaja con un costo objetivo, es decir con un costo máximo referencial, según la afirmación de que los precios de ventas no determinan los costos, lo que implica, que tampoco conoce con anticipación el margen de ganancia de cada uno de los productos, situación que se corresponde con lo reseñado respecto a que desde el punto de vista individual no se conoce el costo de los productos, pero de manera global sí.

La empresa no trabaja con el enfoque justo a tiempo de la compra y la producción. No existen políticas ni metódica definida sobre los procesos de compras de materias primas, materiales de empaques y suministros, lo que origina, con frecuencia, paralización y/o disminución de la producción por falta de materiales y, por consiguiente, en muchas ocasiones, problemas en la entrega de los productos a los clientes en cuanto a cantidad y plazos. En otros momentos, se presentan excesos de materiales y productos terminados en el almacén, convirtiéndose los mismos en capital inmovilizado, lo que trae como consecuencia altos costos de oportunidad y costos de obsolescencia y deterioro, que en PROULA no se determinan y analizan.

Consideran la calidad como factor estratégico. No obstante, la empresa no tiene implantado un sistema de gestión de la calidad, por lo tanto no calculan el costo de la calidad. Pero, si se lleva a cabo un proceso de control de calidad, en lo que respecta a la recepción de materiales, durante el proceso de producción y en los productos terminados. Se trabaja con las buenas prácticas de manufactura (BPM). Sin embargo, en algunas ocasiones se reciben quejas de los clientes por la calidad del producto en cuanto al envasado (derrames de los jarabes). En líneas generales, en PROULA se trabaja con estrictas normas de control de calidad y de sanidad.

Consideramos importante destacar que aunque el departamento predominante para llevar a cabo el proceso de control es el de Calidad, en esta tarea también intervienen la Gerencia de Producción, la Gerencia de investigación y Desarrollo, Laboratorios externos, como son los de las Facultades de Farmacia y de Ciencias de la ULA, y un asesor externo.

La empresa conoce los clientes, zonas y vendedores que otorgan mayor margen de utilidades; así como los productos o líneas de productos que resultan más redituables vender. No obstante, en este punto existen divergencias con respecto a que la empresa no maneja un sistema que permita acumular costos para cada tipo de producto y línea de producción. Además, si sólo se conocen costos globales incurridos en la planta, ¿cómo podría la empresa determinar todos los 
aspectos relacionados con las utilidades referidas a las ventas?

El costo comercial o de marketing se calcula en su globalidad. Lo conforman los costos de personal, depreciación del equipo de reparto, los gastos de promoción y publicidad, las comisiones sobre ventas, los fletes de ventas a terceros en el mercado. Estos costos no se asignan a cada uno de los productos, ni líneas de producción.

La empresa no presupuesta costos para reducir el impacto medioambiental, así como tampoco tiene en cuenta la información en esta materia para la toma de decisiones. Sin embargo, es justo resaltar que pese a que no existe información formalizada, se realizan algunas acciones orientadas hacia la preservación del medioambiente, como por ejemplo: cuando desechan líquidos, pese a que no son nocivos para el ambiente, son diluidos en agua y el recipiente normalmente es quemado (incineran en tanques inactivantes) o roto y depositado en los recipientes destinados para los desechos sólidos. En el caso de las tabletas, éstas también son diluidas en agua y vertidas hacia los sistemas de cloacas; en tanto que la estuchería (folio de aluminio del blíster y la caja) se lleva a unos tanques inactivantes. En cuanto a los gases y vapores, que generalmente se producen el área de control de calidad, porque es allí donde manejan reactivos, se tienen sistemas (campanas) de extracción de gases que minimizan el impacto ambiental.

Siguiendo normas de recomendación emitidas el año 2002 por parte del Ministerio de Sanidad, la empresa tiene previsto realizar algunas acciones para salvaguardar el medio ambiente, que im- plica incurrir en algunos costos para la inactivación de las aguas servidas en el proceso, específicamente en el caso de los antibióticos. Las aguas servidas de la planta van a un sumidero que está localizado en la parte de atrás de la planta, en otra zona, que no es el pozo séptico de las aguas negras. $Y$ a ese sumidero se le tiene que hacer un proceso de inactivación, que consiste en agregar unos componentes químicos para que disuelva y elimine los elementos contaminantes.

\section{Conclusiones}

Aún cuando en el análisis de los resultados ya se han esbozado algunas conclusiones específicas respectos a las prácticas de contabilidad y gestión de costos y otros aspectos importantes con incidencia en los mismos, ejecutadas en la empresa objeto de estudio, se exponen a continuación aquellas que, por su carácter relevante, son dignas de mencionar. De igual forma se formulan algunas recomendaciones, orientadas hacia la excelencia de la empresa.

PROULA es una empresa que en los nueve años que tiene de funcionamiento, ha tenido un crecimiento importante que ha hecho que, en la actualidad, participe activamente en el mercado nacional.

Pese a que en agosto de 2001 recibe la Certificación de Buenas Prácticas de Manufactura (BPM), otorgado por la División de Drogas, Medicamentos y Cosméticos del Ministerio de Salud y Desarrollo Social, con una vigencia de dos años, la empresa no se ha adaptado de manera eficiente a los nuevos avances tecnológicos y no ha establecido las nor- 
mas y procedimientos adecuados que le permitan un mayor y mejor control sobre sus operaciones; determinar los costos de cada uno de los productos que elabora, así como de las distintas líneas de producción (sólidos, líquidos y antibióticos no estériles) y de las diferentes unidades organizacionales (departamentos y gerencias); tomar decisiones estratégicas y tácticas en cuanto a la formulación de políticas globales y planes a largo plazo, desarrollar nuevos productos; entre otros.

Sobre la base de lo anterior, se puede señalar que el sistema contable de la empresa, específicamente lo relacionado a los costos, responde a las exigencias externas de información. Es decir, es una contabilidad de costos orientada hacia las necesidades de la contabilidad financiera (preparación de los estados financieros), para cumplir con las exigencias del fisco nacional, las instituciones financieras, los accionistas, entre otros. Pero no es muy útil desde el punto de vista gerencial, pues al no contar con una buena estructura de costos, se desconocen en cuales se incurre, lo que limita a la empresa a la hora de evaluar y establecer mecanismos de reducción de costos y, en consecuencia, le ocasiona una desventaja frente a sus competidores. Además, la empresa no conoce qué productos son más rentables, qué productos podrían estar generando pérdidas y/o que productos podrían estar subsidiando a otros, pues, la información de costo que se maneja en la misma, es global y no por líneas de producción ni para cada tipo de producto.

El no contar con un sistema de control de inventarios de materiales y de productos terminados, ha hecho que se presenten, en algunos ítems, niveles excesi- vos de los mismos, lo que se traduce en capital inmovilizado para la empresa, que implica un costo de oportunidad que la misma no cuantifica. En otros ítems se han agotado las existencias, lo que ocasiona paralización y/o disminución en los niveles de producción y, en consecuencia, incumplimiento con los clientes. Además, hay productos en existencias con fechas límites de vencimientos que no se pueden depositar en la basura, lo que lleva a incurrir en costos de destrucción.

Creemos que la empresa debe hacer un esfuerzo por implantar un sistema de costos, diseñado en conjunto por los responsables de las áreas de producción, contabilidad de costos, investigación y desarrollo, mercadeo (comercialización y ventas) y gerencia de planta. La naturaleza de las actividades de producción -no orientada a satisfacer especificaciones de clientes, en masa, homogénea por línea de producción, secuencial de un proceso a otro- que se llevan a cabo en la empresa, indican que ésta debe utilizar un sistema de costos por procesos, que permita acumular los costos de materiales, mano de obra y costos indirectos de fabricación, bien por departamentos o por líneas de producción (sólido, antibiótico, líquidos) con base en cierto período de tiempo, que podría ser, entre otros, semanal, mensual, bimensual o trimestral. Además, es aconsejable trabajar con costos objetivos o costos planeados (tarjet costing), porque éstos van a permitir no sólo conocer los costos de cada producto o línea de producto antes de su fabricación y los márgenes de ganancia de cada uno de ellos, sino también evaluar estos costos con miras a establecer mecanismos para su optimización. 
Un aspecto importante de acotar, está referido a los costos comunes de ventas y administración, los cuales para efectos del control de gestión y la toma de decisiones, se deben imputar a los productos, a través de unos criterios o bases de reparto, con el objetivo de determinar el costo total de operación de los mismos y determinar su margen de ganancia. Y, desde el punto de vista contable, a los efectos de preparar los estados financieros externos, considerarlos de acuerdo con las normas y procedimientos contables respectivos, como gastos de ventas y gastos de administración.

Asimismo, a partir del comportamiento de la demanda y los tiempos de entrega por parte de los proveedores, tratar de determinar los niveles mínimos y máximos de los inventarios de materia prima y del material de empaque. De igual forma, tomando en cuenta básicamente el comportamiento del mercado y la capacidad de producción de la planta, intentar fijar el mínimo y máximo de inventario de productos terminados disponibles en el almacén. De esta forma se evita tener, por un lado, los inventarios sobrecargados, los cuales provocan, además de costos administrativos de control y mantenimiento, otros costos y riesgos innecesarios, como consecuencia del espacio que ocupan, deterioros, roturas, robos y obsolescencia, etc. Es importante revisar el tamaño del lote que se fabrica, porque podrían estar elaborando más de lo que determina la demanda. Si bien es cierto que mientras más unidades se fabriquen, los costos fijos unitarios se hacen menores, también es cierto que si no hay una buena rotación del producto se incurre en costos de almacenamiento.

Por otro lado, se evita el quedarse sin stock, que conlleva a una paralización de los procesos e insatisfacción de los clientes. En síntesis, pienso que todo se reduce a un problema de logística. Si se consigue planificar y programar bien las ventas, la producción y las actividades de mantenimiento; comunicar anticipadamente y de forma adecuada las necesidades de producción y de mantenimiento al Departamento de Compras, para que éste genere el proceso necesario ante los proveedores y diseñar unos buenos sistemas de distribución, se puede alcanzar el objetivo de establecer los niveles mínimos y máximo de inventarios.

En cuanto al control de calidad, es recomendable que este se aplique en toda la cadena de valor, comenzando por hacerle un seguimiento al proveedor, pasando por el proceso de recepción de la materia prima, la transformación propiamente dicha, los productos terminados $y$, por último, el servicio de posventa. Un aspecto importante, a tomar en cuenta, es el control de calidad que debe aplicarse a todo el talento humano de la organización. La calidad comienza con el individuo, en sus interacciones con el otro y en todos los procesos que lleva adelante. Se debe formar, capacitar y desarrollar al personal, así como evaluar su desempeño. En fin, se debe capitalizar y gestionar el recurso humano como factor elemental de la productividad de la empresa.

Finalmente, es importante también que la empresa formalice la información de costos y gestión medioambiental, a fin de incorporarlos dentro del presupuesto, la 
contabilidad de costos y la toma de decisiones, pues lo ideal es destinar los costos medioambientales para prevenir el deterioro, en lugar de incurrir en costos mayores por algún daño causado en este sentido.

Se concluye la presente investigación señalando que el diagnóstico y las recomendaciones hechas para las distintas áreas de la organización, se justifican en términos de que todo lo que se haga o, en su defecto, se deje de hacer, genera costos, lo que implica que hay que trabajar teniendo siempre en cuenta que los objetivos deben asegurarse haciendo un uso racional de los recursos, es decir OPTIMIZANDO los COSTOS. Para ello, es necesario que cada uno de los actores de PROULA Medicamentos busque en su ámbito interior la productividad y calidad apropiada a las exigencias de la empresa.

\section{Referencias Bibliográficas}

Alvarez López, José; Amat Salas, Joan; Amat Salas, Oriol; Balada Ortega, Tomás; Blanco Ibarra, Felipe; Castelló Taliani, Emma; Lizcano Álvarez, Jesús y Ripio Feliu, Vicente (1996), Contabilidad de gestión avanzada. Planificación, control y experiencias prácticas. España: McGraw-Hill.

Amat, Oriol y Soldevilla, Pilar (1998), Contabilidad y gestión de costes. Barcelona-España: Ediciones Gestión 2000.

Besterfield, Dale H. (1995), Control de calidad. México: Prentice Hall Hispanoamericana, S.A.

Blanco Ibarra, Felipe (1996), Contabilidad de costos y analítica de gestión para las decisiones estratégicas. Bilbao: Ediciones Deusto, S.A.
Campanella, Jack (1992), Principios de los costes de la calidad. Madrid: Ediciones Díaz de Santos.

Dale, Barrie G. y Plunkett, James J. (1993), Los costos en la calidad. México: Grupo Editorial Iberoamérica.

De la Cuesta, María (1996), El control de gestión en las entidades bancarias. Los modelos tradicionales y el ABM. Madrid: Universidad Nacional de Educación a Distancia.

Fernández, Antonio (1994), Situación actual y perspectivas de la contabilidad de gestión en España. En J. Amat y O. Amat (Coord.), La contabilidad de gestión actual: nuevos desarrollos (pp. 127-142). Asociación Española de Contabilidad y Administración de Empresas (AECA). Madrid: Ediciones Grafica Ortega.

Gayle Rayburn, Leticia (1999), Contabilidad y administración de costos. México: McGraw-Hill Interamericana Editores.

Hansen, Don y Mowen, Maryanne (1996), Administración de costos: contabilidad y control. México: International Thomson Editores, S.A.

Hay, Edward J. (1989), Justo a tiempo (Just in Time). La técnica japonesa que genera mayor ventaja competitiva. Bogotá: Editorial Norma.

Holst, Randolf y Savage, Robert (2002), Herramientas y técnicas para implementar la administración basada en actividades. En S. Player y R. Lacerda (Comp.), Gerencia basada en actividades: Cómo construir una organización más fuerte, más rentable y más efectiva (pp. 3-20). Bogotá: McGraw-Hill Interamericana, S.A.

Horngren, Charles; Foster, George y Datar, Srikant (1996), Contabilidad de costos: Un enfoque gerencial. México: Prentice-Hall Hispanoamericana.

Kaplan, Robert y Cooper, Robin (1999), Coste y efecto: cómo usar el $A B C$, el $A B M$ 
y el ABB para mejorar la gestión, los procesos y la rentabilidad. Barcelona, España: Ediciones Gestión 2000.

Mallo, Carlos; Mir, Fernando; Requena José y Serra, Vicente (1994), Contabilidad de gestión (contabilidad interna): Cálculo, análisis y control de costes para la toma de decisiones. Barcelona, España: Editorial Ariel, S.A.

Mallo, Carlos; Kaplan, Robert; Meljen, Sylvia y Jiménez, Carlos (2000), Contabilidad de costos y estratégica de gestión. Madrid: Prentice Hill lberia.

McCarthy, Jerome y Perreault, Willian D. (1996), Markenting. Madrid: Irwin.

Monden, Yasuhiro (1997), Sistemas de Reducción de Costos. Madrid:TGP Hoshin, Madrid.

Moreno Luzón, María; Peris, Fernando J. y González, Tomás (2001), Gestión de la Calidad y Diseño de Organizaciones: teoría y estudio de casos. Madrid: Prentice-Hall.

Neuner, John J. y Deakin, Edward B. (1997), Contabilidad de Costos: principios y práctica. (Tomo I). México: Editorial Limusa, S.A.

Ramírez Padilla, David (1997), Contabilidad administrativa. México: McGraw-Hill Interamericana Editores, S. A.
Rincón, Haydeé (2000), Sistemas de costos: convencional y actual. Mérida, Venezuela: Facultad de Ciencias Económicas y Sociales de la Universidad de los Andes.

Rincón, Haydeé (2001), Calidad, productividad y costos: análisis de relaciones entre estos tres conceptos. Actualidad Contable Faces. 4(4), 49-61.

Sáez Torrecilla, Angel; Fernández Fernández, Antonio y Gutiérrez Díaz, Gerardo. (1993), Contabilidad de costos y contabilidad de gestión. (Vol. 1). Madrid: McGraw-Hil.

Sakurai, M. (1989), Target costing and how to use it. Journal of cost management. 3 (2), 39-50.

Shank, John y Govindarajan, Vijay (1995), Gerencia estratégica de costos. Bogotá: Editorial Norma.

Suárez, Elsa B. (2001), El efecto de la gestión económica del ente sobre el medio ambiente. Contabilidad y Auditoría. 7 (14), 97-118.

Tanaka, Masayuso; Yoshikawa, Takeo; Innes, John. y Falconer, Mitchell (1997), Gestión moderna de costes. Madrid: Ediciones Díaz de Santos. 